\title{
UTILIZAÇÃo DE REAÇÕES FOTO-FENTON NA PREVENÇÃo DE CONTAMINAÇÕES AGRÍCOLAS
}

\author{
Alam Gustavo Trovó, Ricardo Dalla Villa e Raquel Fernandes Pupo Nogueira* \\ Departamento de Química Analítica, Instituto de Química de Araraquara, Universidade Estadual Paulista, \\ CP 355, 14801-970 Araraquara - SP
}

Recebido em 1/10/04; aceito em 27/1/05; publicado na web em 30/6/05

\begin{abstract}
USE OF PHOTO-FENTON REACTIONS IN THE PREVENTION OF AGRICULTURAL CONTAMINATIONS. In this work the application of the photo-Fenton process for the treatment of washing water of herbicide containers using solar energy was studied. The influence of the $\mathrm{H}_{2} \mathrm{O}_{2}$ concentration and the iron source on the degradation of tebuthiuron and diuron was investigated. The degradation efficiency was strongly affected by the iron source. Using ferrioxalate, total mineralization of diuron and tebuthiuron was obtained either for the individual compounds or for a mixture containing both herbicides, while when using $\mathrm{Fe}\left(\mathrm{NO}_{3}\right)_{3}$, the maximum mineralization reached for both herbicides was only $50 \%$.
\end{abstract}

Keywords: ferrioxalate; herbicides; solar.

\section{INTRODUÇÃO}

A forte dependência de insumos químicos pela agricultura moderna tem sido objeto de inúmeras discussões. Do ponto de vista econômico é praticamente impossível competir com o crescente mercado agrícola internacional sem a utilização dessas substâncias. Além do mais o mercado de biocidas é tão atraente quanto o agrícola, o que estimula seu crescimento. Em 1990, o mercado mundial de biocidas movimentou cerca de 26,4 bilhões de dólares. O Brasil é um dos maiores consumidores mundiais de biocidas, principalmente de herbicidas. As despesas do país com essas substâncias em 2002 foram de cerca de 100 milhões de dólares $^{1}$. Do ponto de vista ambiental, a crescente utilização de insumos químicos poderá acarretar consequiências catastróficas, entre elas a contaminação de água potável, que atualmente é uma grande preocupação mundial ${ }^{2}$. Parte dessa preocupação se deve ao fato de a maior parte dos biocidas produzidos acabarem atingindo a água, seja por deriva durante a aplicação, resíduos de embalagens vazias, lavagem de equipamentos, ou, até mesmo, efluentes de indústrias de biocidas ${ }^{3}$.

Dada a importância comercial da agricultura e da indústria de biocidas, provavelmente o uso extensivo dessas substâncias perdurará ainda por um longo período. Com isso em vista, o mais viável é a utilização racional desses compostos através do controle rigoroso do seu uso e manuseio visando, principalmente, evitar super dosagens, aplicações em locais indevidos e lavagem inadequada de embalagens e equipamentos de aplicação, que muitas vezes é feita nas margens de rios.

O presente trabalho tem como proposta um processo que pode atuar tanto na prevenção da contaminação como na remediação de corpos dágua contaminados por herbicidas. Trata-se do processo foto-Fenton que tem atraído grande interesse devido à sua alta eficiência em gerar radicais hidroxila $\left({ }^{\circ} \mathrm{OH}\right)$ durante a decomposição de $\mathrm{H}_{2} \mathrm{O}_{2}$ catalisada por $\mathrm{Fe}^{2+}$ em meio ácido e irradiação UV-visível $^{4,5}$ (Equações 1 e 2).

$\mathrm{Fe}^{2+}+\mathrm{H}_{2} \mathrm{O}_{2} \rightarrow \mathrm{Fe}^{3+}+{ }^{-} \mathrm{OH}+\cdot{ }^{\circ} \mathrm{OH}$

$\mathrm{Fe}^{3+}+\mathrm{H}_{2} \mathrm{O}+\mathrm{h} v \rightarrow \mathrm{Fe}^{2+}+{ }^{\circ} \mathrm{OH}+\mathrm{H}^{+}$

*e-mail: nogueira@iq.unesp.br
Devido ao seu alto potencial de redução ( $2,8 \mathrm{~V}$ contra um eletrodo normal de hidrogênio), o radical hidroxila consegue oxidar não seletivamente várias classes de compostos orgânicos incluindo inúmeros biocidas ${ }^{6,7}$.

$\mathrm{O}$ processo foto-Fenton tem ainda como vantagens a utilização de reagentes de custos relativamente baixos, sais de $\mathrm{Fe}$ (II) ou (III) e $\mathrm{H}_{2} \mathrm{O}_{2}$, e irradiação solar que é intensa na maior parte do país. Ao final do processo, os íons ferro presentes podem ser precipitados por elevação do $\mathrm{pH}$, enquanto o peróxido de hidrogênio é consumido durante a reação.

A aplicabilidade do processo proposto foi avaliada para o tratamento de água de lavagem de vasilhames dos herbicidas Combine e Herburon, conseguidos junto a um depósito de descarte agrícola que recolhe as embalagens após a tríplice lavagem feita em campo.

No estudo foi avaliada a influência de diferentes fontes de ferro e da concentração de peróxido de hidrogênio. A eficiência do processo de degradação dos compostos nas formulações individualmente foi comparada à eficiência na mistura dos dois herbicidas.

\section{PARTE EXPERIMENTAL}

\section{Reagentes}

Nas determinações cromatográficas foram utilizadas soluções padrão de diuron e tebutiuron (Dr. Ehrenstorfer $\mathrm{GmbH}$ ) e metanol (Mallinckrodt - CLAE)/ácido acético (1\%) (Merck), utilizada como fase móvel. Para as determinações de carbono orgânico total (COT) foram preparados padrões de carbono total, a partir do ftalato ácido de potássio (Nacalai Tesque), e carbono inorgânico, a partir de uma mistura de carbonato e bicarbonato de sódio (Nacalai Tesque). Como fonte de ferro foram utilizados nitrato férrico (Mallinckrodt) e ferrioxalato de potássio $(\mathrm{FeOx})$, que foi preparado a partir do oxalato de potássio (Nuclear) e nitrato férrico (Mallinckrodt) como descrito por Hatchard e Parker ${ }^{8}$. As soluções de $\mathrm{H}_{2} \mathrm{O}_{2}$ utilizadas foram preparadas por diluição de uma solução $30 \%(\mathrm{~m} / \mathrm{v}) \mathrm{H}_{2} \mathrm{O}_{2}$ (Merck). $\mathrm{O} \mathrm{pH}$ das soluções foi ajustado com solução $3 \mathrm{~mol} \mathrm{~L}^{-1} \mathrm{H}_{2} \mathrm{SO}_{4}$ (Synth). 


\section{Compostos estudados}

Neste trabalho foi estudada a fotodegradação de dois herbicidas, diuron [3-(3,4-diclorofenil)-1,1-dimetiluréia] e tebutiuron (TBH) [1-(5-tert-butil-1,3,4-tiadiazol-2-il)-1,3-dimetiluréia] derivados das uréias, e presentes nas formulações comerciais Herburon e Combine, respectivamente. As estruturas moleculares desses compostos são apresentadas na Figura 1.

a<smiles>CN(C)C(=O)Nc1ccc(Cl)c(Cl)c1</smiles>

b<smiles>CNC(=O)N(C)c1nnc(C(C)(C)C)s1</smiles>

Figura 1. Estrutura molecular dos herbicidas: (a) diuron e (b) tebutiuron

As soluções aquosas utilizadas no estudo de fotodegradação foram preparadas por lavagens sucessivas das embalagens dos respectivos herbicidas. Cada embalagem de $5 \mathrm{~L}$ foi lavada três vezes com porções de aproximadamente $0,33 \mathrm{~L}$ de água destilada. Nas soluções resultantes das três lavagens $(\sim 1 \mathrm{~L})$, foram encontradas concentrações de carbono orgânico total (COT) de 30 e 7,0 $\mathrm{mg} \mathrm{L}^{-1}$ e concentrações de 0,23 e $0,028 \mathrm{mmol} \mathrm{L}^{-1}$ dos princípios ativos tebutiuron e diuron, respectivamente.

As embalagens utilizadas no estudo foram conseguidas junto a uma firma de reciclagem de embalagens de pesticidas e já haviam passado pela tríplice lavagem feita em campo.

\section{Condições de irradiação}

Os experimentos foram feitos na cidade de Araraquara, SP $\left(22^{\circ} \mathrm{S}\right.$ $48^{\circ} \mathrm{O}$ ) no mês de julho, sob condições de céu claro em frascos de vidro âmbar com a parte superior aberta $(0,090 \mathrm{~m}$ de diâmetro e $0,045 \mathrm{~m}$ de profundidade de solução), a fim de simular um reator tipo tanque 9 .

As soluções contendo os herbicidas ficaram expostas à irradiação por tempo suficiente para atingir a mesma dose de energia solar para possibilitar melhor comparação dos resultados ${ }^{10}$, sendo a dose de energia a integral da intensidade da irradiação com o tempo (Equação 3):

Dose de energia $\left(\mathrm{J} \mathrm{cm}^{-2}\right)=\int_{t_{1}}^{t_{2}}$ intensidade luminosa $\left(\mathrm{W} \mathrm{cm}^{-2}\right) \mathrm{xdt}(\mathrm{s})(3)$

A intensidade luminosa e dose de energia solar foram medidas por um radiômetro PMA 2100 (Solar Light CO) na região UV-A (320 a $400 \mathrm{~nm}$ ) com sensor na posição horizontal. Antes dos experimentos, o pH da solução dos herbicidas foi ajustado para $2,5 \mathrm{com}$ solução 3,0 mol L-1 $\mathrm{H}_{2} \mathrm{SO}_{4}$, $\mathrm{pH}$ ótimo segundo trabalho anterior ${ }^{11}$. Imediatamente antes da exposição à luz solar foi adicionado ferrioxalato de potássio ou nitrato férrico e peróxido de hidrogênio.

\section{Análises químicas}

A eficiência do processo de mineralização, conversão do carbono orgânico a $\mathrm{CO}_{2}$, foi avaliada pela medida do decaimento da concentração de carbono orgânico total (COT), utilizando um analisador de carbono (TOC-5000A-Shimadzu). Estas determinações foram feitas imediatamente depois da retirada das amostras do reator, evitando-se assim reações posteriores. O carbono orgânico total determinado nestas soluções não é proveniente apenas dos herbicidas. Produtos formados na sua degradação e outros compostos que estão presentes na formulação bem como o oxalato do ferrioxalato de potássio são fontes de carbono orgânico total. A porcentagem de mineralização para uma determinada dose de energia foi calculada em relação à concentração de carbono orgânico inicial presente na amostra.

A concentração de diuron e tebutiuron durante a fotodegradação foi monitorada por cromatografia líquida de alta eficiência (CLAE), utilizando-se um equipamento Varian ProStar 230 com detector UV-Vis ProStar 310, coluna Phenomex $5 \mu$ C-18 (0,25 x 0,0046 m). A porcentagem de oxidação dos herbicidas para uma determinada dose de energia foi calculada em relação à concentração inicial dos herbicidas. Os herbicidas foram extraídos da fase aquosa por extração em fase sólida utilizando cartuchos Sep-Pak C-18 Waters. Nestas extrações, $2,5 \mathrm{~mL}$ de amostra aquosa foram aplicados aos cartuchos que foram previamente condicionados com 5,0 mL de metanol, com uma seringa de vidro, a uma vazão de 5,0 $\mathrm{mL} \mathrm{min}^{-1}$. Após as extrações, os cartuchos foram armazenados em geladeira a $5{ }^{\circ} \mathrm{C}$. Instantes antes das determinações cromatográficas os analitos foram eluidos com $2,5 \mathrm{~mL}$ de metanol. As condições cromatográficas utilizadas para a determinação de tebutiuron e diuron na mistura foram: vazão $1,0 \mathrm{~mL} \mathrm{~min}^{-1}$, comprimento de onda de detecção de 254 nm e metanol:ácido acético 1\% (60:40) como fase móvel. Para as amostras provenientes de degradação de somente diuron, a fase móvel utilizada foi metanol:ácido acético 1\% (70:30) a fim de diminuir o tempo de análise. Foram feitos testes de recuperação para ambos herbicidas em solução aquosa, obtendo-se recuperações de 82 a $110 \%$ para as fortificações de 0,01 e $0,1 \mathrm{mmol}$ $\mathrm{L}^{-1}$, respectivamente.

\section{RESULTADOS E DISCUSSÃO}

\section{Influência da concentração de $\mathrm{H}_{2} \mathrm{O}_{2}$}

A fim de avaliar a influência da concentração de $\mathrm{H}_{2} \mathrm{O}_{2}$ no tratamento da água de lavagem de vasilhames dos herbicidas diuron e tebutiuron, foram feitos experimentos de fotodegradação em concentrações iniciais de $\mathrm{H}_{2} \mathrm{O}_{2}$ de 10 e $20 \mathrm{mmol} \mathrm{L}^{-1}$. Como fonte de ferro foi inicialmente utilizada solução $1,0 \mathrm{mmol} \mathrm{L}^{-1}$ de nitrato férrico (Figura 2).

Na Figura 2a pode ser observado, pelos resultados obtidos por CLAE, que para uma dose de energia de $0,58 \mathrm{~J} \mathrm{~cm}^{-2}$ (aproximadamente 5 min de irradiação) o herbicida diuron foi totalmente oxidado em ambas as concentrações de $\mathrm{H}_{2} \mathrm{O}_{2}$ utilizadas. Para o tebutiuron, mesmo em maiores doses de energia, a oxidação não ultrapassou $20 \%$. Provavelmente uma das causas da menor porcentagem de oxidação do tebutiuron é que sua concentração inicial em solução é aproximadamente sete vezes maior que a concentração inicial do diuron.

Os resultados obtidos por CLAE indicam a oxidação do composto-alvo, no entanto, não dão informações sobre a mineralização do composto. Este tipo de informação é importante, pois o composto pode estar sendo oxidado, porém não mineralizado, formando produtos de toxicidade desconhecida. Sendo assim, foram feitas determinações de COT, visando avaliar não apenas a oxidação, mas também a mineralização dos herbicidas. Pode ser observado na Figura 2 b que o aumento da concentração de peróxido de hidrogênio de 10 para $20 \mathrm{mmol} \mathrm{L}^{-1}$ não teve influência significativa na 

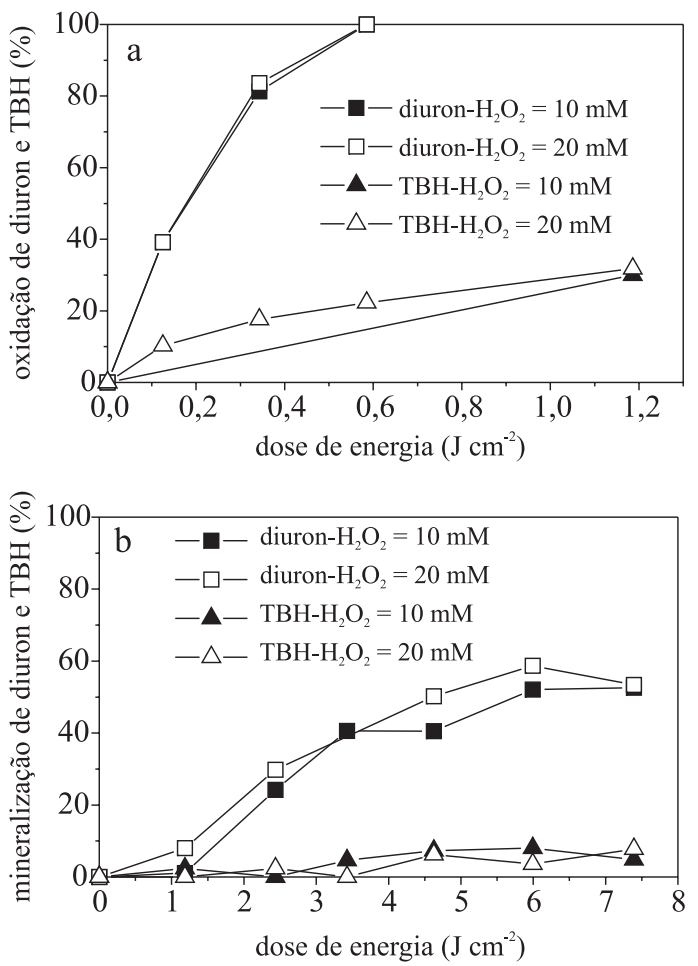

Figura 2. Influência da concentração inicial de $\mathrm{H}_{2} \mathrm{O}_{2}$ na (a) oxidação de diuron e tebutiuron e $(b)$ mineralização de diuron e tebutiuron. Concentrações iniciais: $\left[\mathrm{Fe}\left(\mathrm{NO}_{3}\right)_{3}\right]=1,0 \mathrm{mmol} \mathrm{L}{ }^{-1}$; [diuron] $=0,028 \mathrm{mmol} \mathrm{L}^{-1}$ e $[\mathrm{COT}]=$

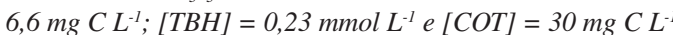

mineralização dos herbicidas. Em ambas as concentrações e a uma mesma dose de energia de $7,4 \mathrm{~J} \mathrm{~cm}^{-2}$ obteve-se 50 e $8,0 \%$ de mineralização para diuron e tebuthiuron respectivamente. Parra e colaboradores $^{12}$ também observaram que a variação da concentração de $\mathrm{H}_{2} \mathrm{O}_{2}$ não tem grande influência na degradação de outros herbicidas em meio aquoso. Comparando os resultados apresentados nas Figuras 2a e $2 \mathrm{~b}$ observa-se que apesar do diuron ter sido $100 \%$ oxidado, atingiu porcentagem de mineralização de somente $50 \%$ na presença de $\mathrm{Fe}\left(\mathrm{NO}_{3}\right)_{3}$ como fonte de ferro, o que indica a presença de intermediários de degradação.

Experimentos feitos na ausência de irradiação solar e presença de $\mathrm{Fe}\left(\mathrm{NO}_{3}\right)_{3}$ e $\mathrm{H}_{2} \mathrm{O}_{2}$ indicaram que $7 \%$ de tebutiuron são oxidados, não havendo mineralização. Nas mesmas condições, houve $48 \%$ de oxidação e $30 \%$ de mineralização de diuron. Essa degradação provavelmente ocorre devido à geração do radical hidroperoxila, a partir da reação de $\mathrm{Fe}^{3+}$ com $\mathrm{H}_{2} \mathrm{O}_{2}$ conforme Equações 4 e $5^{4}$, que é menos reativo que o radical hidroxila.

$\mathrm{Fe}^{3+}+\mathrm{H}_{2} \mathrm{O}_{2} \rightarrow \mathrm{Fe}-\mathrm{OOH}^{2+}+\mathrm{H}^{+} \mathrm{k}=0,001-0,01 \mathrm{M}^{-1} \mathrm{~s}^{-1}$

$\mathrm{Fe}-\mathrm{OOH}^{2+} \rightarrow \mathrm{HO}_{2}^{\cdot}+\mathrm{Fe}^{2+}$

Na presença de irradiação e ausência de reagentes não houve degradação dos compostos, indicando que não ocorre a fotólise direta destes herbicidas sob luz solar. Na ausência de ferro e presença de $\mathrm{H}_{2} \mathrm{O}_{2}$ e irradiação solar, $8 \%$ de diuron foram oxidados, não havendo oxidação de tebutiuron. $\mathrm{Na}$ presença de $\mathrm{Fe}\left(\mathrm{NO}_{3}\right)_{3} \mathrm{e}$ irradiação solar, 11 e $20 \%$ de tebutiuron e diuron foram oxidados, respectivamente, não havendo mineralização de nenhum desses compostos. Neste caso, a oxidação dos compostos pode ser atribuída à geração de radicais hidroxila pela hidrólise do $\mathrm{Fe}^{3+}$ na presença de irradiação (Equação 2).

Estes resultados indicam que a reação foto-Fenton é a princi- pal responsável pela degradação dos herbicidas, considerando as baixas degradações obtidas quando $\mathrm{H}_{2} \mathrm{O}_{2}$, ferro e irradiação solar foram avaliados separadamente.

\section{Influência da fonte de ferro na fotodegradação dos herbicidas diuron e tebutiuron}

Nos experimentos anteriores foi observado que a variação da concentração de $\mathrm{H}_{2} \mathrm{O}_{2}$ não influenciou significativamente a fotodegradação dos contaminantes (Figura 2). Considerou-se então que a mudança da fonte de ferro pudesse favorecer a degradação, como verificado por Safarzadeh- Amiri ${ }^{13,14}$ para outros compostos orgânicos. Foram feitos experimentos utilizando FeOx na concentração inicial de $1,0 \mathrm{mmol} \mathrm{L}^{-1}$ e solução $10 \mathrm{mmol} \mathrm{L}^{-1}$ de $\mathrm{H}_{2} \mathrm{O}_{2}$. A Figura 3 apresenta os resultados desses experimentos comparando-os com os apresentados na Figura 2.
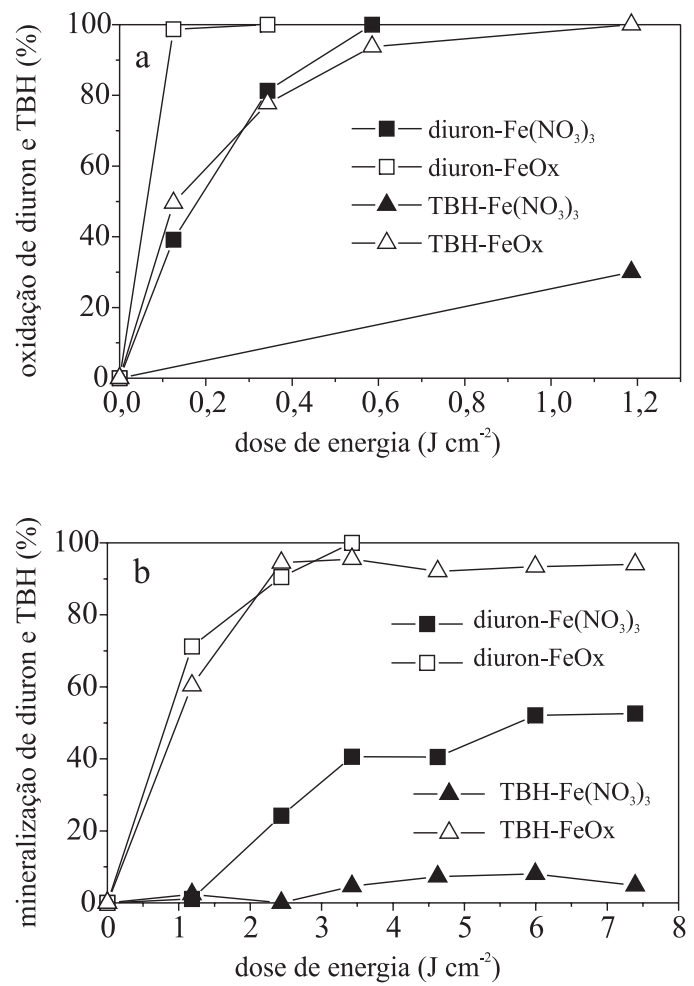

Figura 3. Influência da fonte de ferro na (a) oxidação de diuron e tebutiuron $e$ (b) mineralização de diuron e tebutiuron. Concentrações iniciais: $\left[\mathrm{Fe}\left(\mathrm{NO}_{3}\right)_{3}\right.$ ou FeOx] = 1,0 mmol L-1; [diuron] = 0,028 $\mathrm{mmol} \mathrm{L}^{-1}$ e [COT] = 6,6 $\mathrm{mg} \mathrm{CL^{- }}$ ${ }^{1} \mathrm{com} \mathrm{Fe}\left(\mathrm{NO}_{3}\right)_{3}$ e $79 \mathrm{mg} \mathrm{CL} L^{-1} \mathrm{com} \mathrm{FeOx}$; $[\mathrm{TBH}]=0,23 \mathrm{mmol} \mathrm{L}^{-1}$ e [COT] $=$ $30 \mathrm{mg} \mathrm{CL}^{-1} \mathrm{com} \mathrm{Fe}\left(\mathrm{NO}_{3}\right)_{3}$ e $102 \mathrm{mg} \mathrm{CL}^{-1} \mathrm{com} \mathrm{FeOx}$

Nos experimentos utilizando-se FeOx, obteve-se $100 \%$ de oxidação do diuron com uma dose de energia de $0,12 \mathrm{~J} \mathrm{~cm}^{-2}$ (1 min de irradiação). Essa mesma porcentagem foi atingida para o tebutiuron, porém com uma maior dose de energia, $1,2 \mathrm{~J} \mathrm{~cm}^{-2}(10$ min) (Figura 3a). Com relação à mineralização (Figura 3b), quando se usa $\mathrm{FeOx}$ e uma dose de energia de $3,4 \mathrm{~J} \mathrm{~cm}^{-2}$ é atingida, o diuron e o tebutiuron são quase totalmente mineralizados. Na presença de $\mathrm{Fe}\left(\mathrm{NO}_{3}\right)_{3}$ e nesta mesma dose de energia, a porcentagem de mineralização atingida foi de $40 \%$ para o diuron e de $3,0 \%$ para o tebutiuron. A comparação desses resultados evidencia a maior velocidade de degradação quando o $\mathrm{FeOx}$ é utilizado como fonte de ferro. Safarzadeh-Amiri e colaboradores ${ }^{13,14}$ também observaram que o $\mathrm{FeOx}$ é mais eficiente que o nitrato férrico na degradação de tolueno e vários outros compostos orgânicos. 
Devido à presença de pares de elétrons não compartilhados nos átomos de nitrogênio, enxofre e grupo carboxila presentes nas estruturas dos compostos estudados, possivelmente quando se utiliza nitrato férrico há formação de complexos entre o ferro e os herbicidas, impedindo a reação destes íons com $\mathrm{H}_{2} \mathrm{O}_{2}$ na reação de Fenton. A fotólise do $\mathrm{FeOx}$ resulta na geração contínua de $\mathrm{Fe}^{2+}$ com alto rendimento quântico ${ }^{14}$ (Equações 6 e 7) que reage com o peróxido aumentando a geração de ${ }^{\circ} \mathrm{OH}$. Isso explica a melhor eficiência de degradação dos herbicidas quando o nitrato férrico é substituído por $\mathrm{FeOx}$.

$$
\begin{gathered}
{\left[\mathrm{Fe}\left(\mathrm{C}_{2} \mathrm{O}_{4}\right)_{3}\right]^{3-}+\mathrm{h} v \rightarrow \mathrm{Fe}^{2+}+2 \mathrm{C}_{2} \mathrm{O}_{4}^{2-}+\mathrm{C}_{2} \mathrm{O}_{4}^{-\bullet}} \\
\mathrm{C}_{2} \mathrm{O}_{4}^{-\bullet}+\left[\mathrm{Fe}\left(\mathrm{C}_{2} \mathrm{O}_{4}\right)_{3}\right]^{3-} \rightarrow \mathrm{Fe}^{2+}+3 \mathrm{C}_{2} \mathrm{O}_{4}^{2-}+2 \mathrm{CO}_{2}
\end{gathered}
$$

Novamente, para avaliar o efeito de algumas variáveis do processo de fotodegradação foram feitos controles experimentais e constatou-se que no escuro e na presença de $\mathrm{FeOx}$ e $\mathrm{H}_{2} \mathrm{O}_{2}$ não ocorre oxidação dos herbicidas, após 30 min de experimento. Na ausência de $\mathrm{H}_{2} \mathrm{O}_{2}$ e na presença de irradiação e $\mathrm{FeOx}$, obteve-se $13 \%$ de oxidação e $12 \%$ de mineralização de tebutiuron. Nestas mesmas condições, $23 \%$ de mineralização e $51 \%$ de oxidação foram obtidos para o diuron. Estas porcentagens podem ser justificadas tanto pela presença de radicais hidroxilas, gerados pela hidrólise do $\mathrm{Fe}^{3+}$ (Equação 2), quanto pelos radicais oxalato gerados durante a fotólise do $\mathrm{FeOx}$ (Equação 6).

\section{Aplicação do processo foto-Fenton/solar para tratamento de uma amostra contendo a mistura dos herbicidas diuron e tebutiuron}

Uma vez que a combinação de formulações é uma prática comum na agricultura, foram feitos experimentos de fotodegradação envolvendo a mistura dos herbicidas diuron e tebutiuron. Tendo em vista a importância da fonte de ferro na degradação dos compostos individuais, foram feitos experimentos para avaliar sua influência na degradação da mistura de diuron e tebutiuron. Nestes foram utilizados nitrato férrico e $\mathrm{FeOx} 1,0 \mathrm{mmol} \mathrm{L}^{-1}$ e $10 \mathrm{mmol} \mathrm{L}^{-1}$ de $\mathrm{H}_{2} \mathrm{O}_{2}$. Os resultados destes experimentos são apresentados na Figura 4.

Como pode ser observado na Figura 4a, elevadas porcentagens de oxidação de diuron e tebutiuron na mistura ocorrem mesmo em baixas doses de energia quando $\mathrm{FeOx}$ é utilizado como fonte de ferro. Com nitrato férrico também foi possível obter total oxidação de diuron, mas não de tebutiuron para a mesma dose de energia aplicada. O aumento da porcentagem de oxidação do tebutiuron, na presença de nitrato férrico com a dose de energia, apresentado na Figura 4a, sugere que uma maior dose de energia pode levar à oxidação total deste composto. A mineralização dos componentes da mistura na presença de $\mathrm{FeOx}$ também foi mais eficiente que na presença de $\mathrm{Fe}\left(\mathrm{NO}_{3}\right)_{3}$. Com uma dose de energia de $2,4 \mathrm{~J} \mathrm{~cm}^{-2}$ (20 min) obteve-se $84 \%$ de mineralização dos herbicidas, enquanto que com nitrato férrico o valor atingido foi de $8 \%$ (Figura 4b). Estes resultados demonstram uma alta eficiência do processo foto-Fenton utilizando $\mathrm{FeOx}$, mesmo na mistura de herbicidas.

\section{Potencial de aplicação}

Em 20 min de irradiação solar, em dias de inverno, obtevese $84 \%$ de mineralização da mistura de diuron e tebutiuron, utilizando-se um reator de $0,090 \mathrm{~m}$ de diâmetro, 0,045 $\mathrm{m}$ de profundidade de solução e 0,27 L de solução. Nogueira e colaboradores $^{9}$ relataram que estes resultados podem ser aumentados em
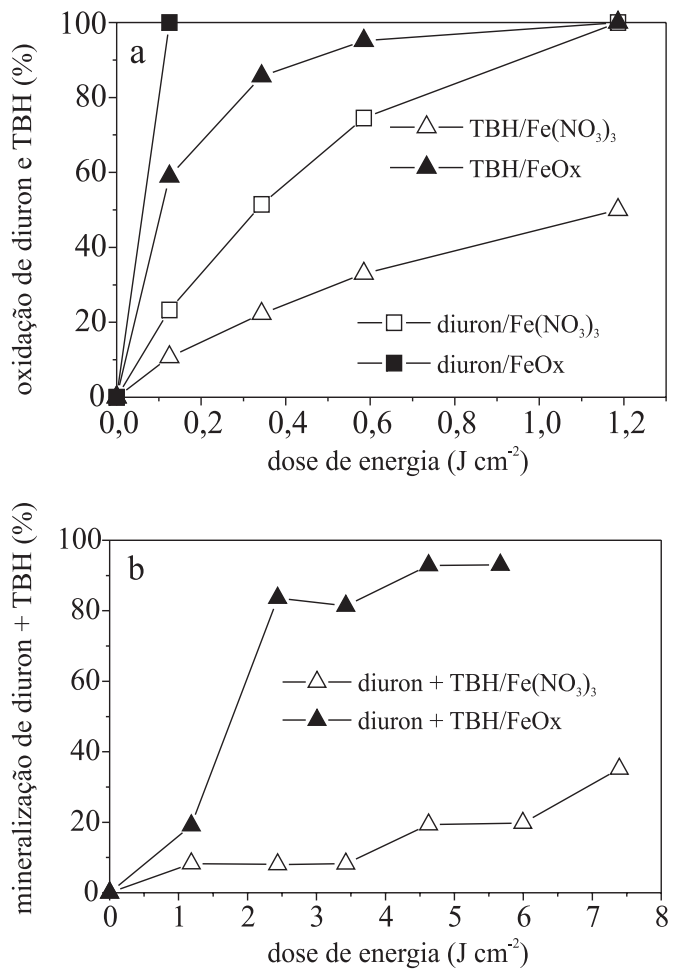

Figura 4. Comparação da eficiência de (a) oxidação e (b) mineralização durante a fotodegradação da mistura dos herbicidas diuron e tebutiuron com diferentes fontes de ferro $\left(\mathrm{Fe}\left(\mathrm{NO}_{3}\right)_{3}\right.$ ou $\left.\mathrm{FeOx}=1,0 \mathrm{mmol} \mathrm{L}^{-1}\right)$, na concentração inicial de $10 \mathrm{mmol} \mathrm{L}^{-1}$ de $\mathrm{H}_{2} \mathrm{O}_{2}$

até $40 \%$ durante o verão. Isso permite estimar que um sistema em maior escala como um tanque de profundidade de $0,045 \mathrm{~m}$, com uma área relativamente pequena, da ordem de $10 \mathrm{~m}^{2}$, seria suficiente para tratar uma vazão de água contaminada com mistura de diuron e tebutiuron de aproximadamente $15 \mathrm{~m}^{3} / \mathrm{dia}$ no verão (10 h de irradiação) e 7,0 $\mathrm{m}^{3} /$ dia no inverno ( $8 \mathrm{~h}$ de irradiação). Considerando-se que três lavagens com 0,33 L são suficientes para remover a maior parte dos herbicidas presentes nas embalagens, um reator com as dimensões acima teria capacidade para tratar a água usada na lavagem de aproximadamente 15 mil embalagens com capacidade para 5,0 L, em um só dia e as embalagens poderiam ser destinadas à reciclagem, livres de contaminação.

Outra vantagem desse tratamento é a degradação não seletiva dos contaminantes, permitindo a mineralização de mistura de formulações, não havendo necessidade de separação das embalagens. No final do processo, o peróxido de hidrogênio é totalmente consumido e os íons férricos podem ser precipitados por elevação do $\mathrm{pH}$ antes do descarte.

\section{Procedimento para aplicação}

Supondo-se que o processo proposto venha a ser aplicado na remediação de água contaminada com herbicidas nos níveis de concentração estudados, descrevem-se a seguir os passos para tratamento de um volume compatível com as dimensões do reator citado no item Potencial de aplicação $\left(10 \mathrm{~m}^{2}\right.$ x 0,060 m):

- construir um tanque com as dimensões acima, em local exposto à luz solar;

- completar o volume do tanque até uma altura de 0,045 m com água contaminada proveniente da lavagem de vasilhames ou de outro utensílio agrícola;

- adicionar $250 \mathrm{~g}$ de ferrioxalato e homogeneizar a solução; 
- ajustar o pH para 2,5 (o que pode ser feito com ácido sulfúrico e uso de papel indicador universal);

- adicionar de $0,5 \mathrm{~L}$ de $\mathrm{H}_{2} \mathrm{O}_{2}(30 \% \mathrm{~m} / \mathrm{v})$ com o sistema sob irradiação solar (preferencialmente no período das 10 às $15 \mathrm{~h}$ ).

\section{Observações:}

- com base nos resultados aqui obtidos estima-se que em um tempo de $30 \mathrm{~min}$ os herbicidas tenham sido completamente mineralizados.

- a geração de ferrioxalato pode ser feita in situ ${ }^{15,17}$, pela adição de $121 \mathrm{~g}$ de nitrato férrico e $250 \mathrm{~g}$ de oxalato de potássio.

\section{CONCLUSÕES}

A simplicidade e o baixo custo são características importantes do processo proposto e viabilizam sua aplicação no tratamento de água de lavagem de frutas, verduras, equipamentos de aplicação de biocidas e na remediação de corpos d'água já contaminados. Esse depende basicamente do $\mathrm{pH}$ da solução, irradiação solar, peróxido de hidrogênio e da fonte ferro, que foi a variável de maior influência no processo. Quando o $\mathrm{FeOx}$ foi utilizado obteve-se aproximadamente $100 \%$ de mineralização, tanto para os herbicidas individuais quanto para a mistura dos mesmos, o que evidencia a potencialidade de aplicação do processo fotoFenton sob luz solar para tratamento de águas contaminadas com estes compostos.

\section{AGRADECIMENTOS}

À FAPESP por financiar este trabalho (Processos no. 02/007379 e 02/11056-2) e à Profa. Dra. M. V. Boldrin por disponibilizar o cromatógrafo.

\section{REFERÊNCIAS}

1. http://www.desenvolvimento.gov.br/arquivo/sdp/proAcao/ forCompetitividade/docInstalacoes/dialndAgroquimica.pdf, acessada em Agosto 2004.

2. Romero, A. H. H.; Hernández, C. T.; Malo, E. A.; Mendoza, R. B.; Mar. Pollut. Bull. 2004, 48, 1130.

3. Malato, S.; Caceres, J.; Aguera, A.; Mezcua, M.; Hernando, D.; Vial, J.; Fernández-Alba, A. R.; Environ. Sci. Technol. 2001, 35, 4359.

4. Neyens, E.; Baeyens, J.; J. Hazard. Mater. 2003, 98, 33.

5. Pignatello, J. J.; Environ. Sci. Technol. 1992, 26, 944.

6. Legrini, O.; Oliveros, E.; Braun, A. M.; Chem. Rev. 1993, 93, 671.

7. Huang, C. P.; Dong, C.; Tang, Z.; Waste Management 1993, 13, 361.

8. Hatchard, C. G.; Parker, C. A.; Proc. Royal Soc. 1956, A235, 518.

9. Nogueira, R. F. P.; Trovó, A. G.; Modé, D. F.; Chemosphere 2002, 48, 385.

10. Nogueira, R. F. P.; Trovó, A. G.; Paterlini, W. C.; Water Sci. Technol. 2004, 49, 195.

11. Nogueira, R. F. P.; Guimarães, J. R.; Water Res. 2000, 34, 895.

12. Parra, S.; Sarria, V.; Malato, S.; Péringer, P.; Pulgarin, C.; Appl. Catal., B 2000, 27, 153 .

13. Safarzadeh-Amiri, A.; Bolton, J. R.; Cater, S. R.; J. Adv. Ox. Technol. 1996, 1,8 .

14. Safarzadeh-Amiri, A.; Bolton, J. R.; Cater, S. R.; Sol. Energy 1996, 56, 439 .

15. Emilio, C. A.; Jardim, W. F.; Litter, M. I.; Mansila, H. D.; J. Photochem. Photobiol., A 2002, 151, 121.

16. Aplin, R.; Feitz, A. J.; Waite, T. D.; Water Sci. Technol. 2001, 44, 23.

17. Mazellier, P.; Sulzberger, B.; Environ. Sci. Technol. 2001, 35, 3314. 